\title{
Antibacterial resistance via GyrB24 targeting
}

\begin{abstract}
In nature, four mutations confer resistance to coumarins in Escherichia coli strains: Arg 136 to Cys, His or Ser and Gly-164 to Val. All of these mutations are located at the ATP-binding site for the B subunit of the gyrase enzyme. GyrB proteins with these mutations losses their enzymatic activity. Molecular visualizations showed that Arg-136 in gyrB24 is hydrogen bounded through its guanidinium group to the two oxygen atoms in the Coumarin ring. Planning of new compounds with more profound antibiotic activities includes extending the alkyl group at the 5 position of the noviose sugar for the Coumarin. On the other hand, more studies are needed to assess the molecular interactions between comuarins and the host cells to identify specific moieties involved in allergy (jaundice) reactions evoked by the antibiotics.
\end{abstract}

Volume 4 Issue I - 2017

\author{
Ali Bin Thani \\ Department of Biology, University of Bahrain, Bahrain
}

Correspondence: Ali Bin Thani, Department of Biology, College of Science, University of Bahrain, Bahrain, Tel +97317437419, Email abinthani@uob.edu.bh

Received: October 16, 2016 | Published: March 03, 2017

\section{Abbreviations: UPEC, uropathogenic escherichia coli}

\section{Introduction}

In Escherichia coli, the gyrase enzyme is composed of two subunits: A and B. ${ }^{1}$ The A subunit $(97 \mathrm{k} \mathrm{Da})$ interacts and relieves DNA super coils through its active site (tyrosine) during DNA replication. The B subunit contains the ATP as active site required to release the energy in the attached ATP molecule and provides the free energy to the reaction (DNA super coiling) accomplished by the gyrase enzyme. ${ }^{2}$ Amino coumarins/coumarins such as novo biocin and coumermycin A1 are associated with a $24-\mathrm{kDa}$ (residues 2-220) sub-domain in the B subunit of the gyrase enzyme known as amino terminal. ${ }^{3}$ This association overlaps the ATP as site in gyro Band prevents further binding of ATP molecules. ${ }^{4-7}$ However, the poor activity of aminocoumarinsas inhibitors for Gram-negative bacteria, their cytotoxic effects on mammalian cells and low water solubility reduced their use in clinical practice. ${ }^{8}$ On the other hand, new studies are investigating the possibility of emulating the mode of action of coumarins using new molecules with less toxic effects and better inhibitory reactions. ${ }^{8}$ In nature, four mutations confer resistance to coumarins in Escherichia coli strains: Arg-136 to Cys, His or Her and Gly-164 to Val. ${ }^{9-12}$ All of these mutations are located at the ATP-binding site for the B subunit. ${ }^{9-14} \mathrm{GyrB}$ proteins with these mutations losses their enzymatic activity. ${ }^{9}, 15$ Other investigators introduced site-directed mutations at amino acids interacting with aminocoumarinsas. The mutations were able to reduce binding of protein with aminocoumarinsas but at the same time were unable to bind ATP as well. The introduced mutations were Asp73 to Asp and Asn46 to Asp and Leu in GyrB of E. Coli spp. ${ }^{16}$ Other investigators introduced the following site-directed mutations in gyrB: Asp73 (to Glu), Gly77 (to Ala and Ser), Ile78 (to Ala and Leu), and Thr165 (to Ala and Val). ${ }^{15}$ They observed that the E. coli mutants were resistant to novobiocin but with clear loss of enzymatic activity. ${ }^{15}$ One important aspect noted during the study of these mutations regarding the size of the interacting molecule. Studies indicate that the number of produced mutations is remarkably linked to the size of the drug. For example, cyclothialidines (Antibiotic family with similar properties as aminocoumarinsas, targeting the ATP ase site of $g y r \mathrm{~B}$, but usually are larger) are known to complex and overlap regions of the gyrase B subunit as aminocoumarinsas. However, the type of interaction made by cyclothialidines and coumermycin appears to be more profound with more contact areas on the gyrase B subunit than is the case with novobiocin. For example, investigators noted that a single mutation could confer resistance to high concentrations of novobiocin. While, a remarkable decrease in bacterial susceptibility to both cyclothialidines and coumermycin inis associated with double mutations at the ATPase site. The reference attributed the case above to the "bulkier molecular nature" of coumermycin and cyclothialidine compared tonovobiocin. ${ }^{17}$

\section{Effects of gyrase mutations on the expression of virulence genes in pathogenic strains}

Due to the importance of gyrase enzyme for both DNA replication and gene expression, it is expected that the expression of certain core genes will be affected by the mutations that reduce the enzyme activity. Mutations in the gyrA gene of Uropathogenic Escherichia coli UPEC are known to cause a decrease in the virulence of pathogenic bacteria due to their effects on DNA super coiling and expression of several virulence factors. ${ }^{18}$ Such UPEC strains showeda decreasing capacity to cause cystitis and pyelonephritis. ${ }^{18}$ However, no similar studies support a similar finding for mutations in $\operatorname{gyr} B$ and coumarin resistance.

\section{Discussion}

The catalytic ATPase site of gyrB is a $24 \mathrm{KDa}$ fragment. This site is target for most known coumarins. ${ }^{19-21}$ Four chemical groups are making the coumarin: L-noviose sugar, the 5-methylpyrrole-2carboxylic acid, the 3-aminocoumarin ring, and the 4-hydroxy-3isoprenylbenzoyl moiety. ${ }^{19,20}$ Molecular structure analysis showed that Arg-136 in gyrB 24 is hydrogen bounded through its guanidinium group to the two oxygen atoms in the coumarin ring. ${ }^{19,20}$ Mutations that involves removal of Arg-136 contributes to the entropy introduced by ordered water molecule sequestering into the volume vacated by the removed amino acid. ${ }^{19,20}$ Actually ordered water molecules surrounding the ATPase site has mediated hydrogen bonds linking antibiotics such as novobiocin to the mutant molecule. ${ }^{19}$ Usually favorable enthalpic changes are introduced by such interactions and significant changes to heat capacity of the system were monitored. ${ }^{19}$ Studies showed that both hydroxyl benzoate isopentenyl moiety and the 5'-methyl group of the noviose have a stabilization effect on the formation of the complex..$^{19}$ Planning of new compounds with more profound antibiotic activities include extended alkyl group at the $5^{\prime}$-position of the noviose sugar. ${ }^{19-21}$ Thus, both methyl groups of 
the noviose sugar are contributing to the hydrophobic interactions with the following residues in the ATPase site: Ile 78 and Ile90. The remaining 5-methylpyrrole ring is also involved in hydrophobic pocket formations within gyrB24 with the following interaction points Val43, Ala47, Val71, Ile78, Val120, and Val167. ${ }^{19-21}$ The above information represent the importance of key residues in the hydrophobic interactions between coumarins (inhibitors) and the $24 \mathrm{kDa}$ ATPase site and that in the absence of the antibiotic these hydrophobic pockets are occupied with trapped water molecules. Understanding the molecular basis for the interactions between the antibiotics and their target sites would enable better adjustments to enhance activity and affinity of the antibiotics. From a literature point view it looks that more studies are needed to assess the molecular interactions between comuarins and the host cells to identify specific moieties involved in allergy (jaundice) reactions evoked by the antibiotics. ${ }^{19-21}$

\section{Conclusion}

GyrB mutations rendering the bacterial cells resistant to aminocoumarinsas are also involved in deactivating the enzyme. The number of mutations introduced depends on the size of the molecule. Studies showed that water molecules surrounding the gyrB24 protein will affect the enthalpy of the molecules interacting. Future design of coumarins-like drugs could use the extended alkyl groups attaching to the noviose sugar to enhance the activity of the antibiotic. Other parts of the coumarin (e.g., 4-hydroxy-3-isoprenylbenzoyl moiety) are supporting the hydrophobic nature of the antibiotic by folding back onto the coumarin ring, thus, avoiding the hydrophilic residues of the ATPase.

\section{Acknowledgements}

None.

\section{Conflict of interest}

Author declares that there is no conflict of interest.

\section{References}

1. Champoux JJ. DNA topoisomerases: structure, function, and mechanism. Annu Rev Biochem. 2001;70:369-413.

2. Reece RJ, Maxwell A. DNA gyrase: structure and function. CRC Crit Rev Biochem Mol Biol. 1991;26(3-4):335-375.

3. Gilbert EJ, Maxwell A. The $24 \mathrm{kDa} \mathrm{N}$-terminal sub-domain of the DNA gyrase B protein binds coumarin drugs. Molec Microbiol. 1994;12(3):365-373.

4. Lewis RJ, Singh OMP, Smith CV, et al. The nature of inhibition of DNA gyrase by the coumarins and the cyclothialidines revealed by X-ray crystallography. EMBO J. 1996;15(6):1412-1420.

5. Mizuuchi K, O’Dea MH, Gellert M. DNA gyrase: subunit structure and ATPase activity of the purified enzyme. Proc Natl Acad Sci USA. 1978;75(12):5960-5963.

6. Sugino A, Cozzarelli NR. The intrinsic ATPase of DNA gyrase. J Biol Chem. 1980;255(13):6299-6306.
7. Sugino A, Higgins NP, Brown PO, et al. Energy coupling in DNA gyrase and the mechanism of action of novobiocin. Proc Natl Acad Sci USA. 1978;75(10):4838-4842.

8. Collin F, Karkare S, Maxwell A. Exploiting bacterial DNA gyrase as a drug target: current state and perspectives. Appl Microbiol Biotechnol. 2011;92(3):479-497.

9. Contreras A, Maxwell A. gyrB mutations which confer coumarinresistance also affect DNA super coiling and ATP hydrolysis by Escherichia coliDNA gyrase. Mol Microbiol. 1992;6(12):1617-1624.

10. Del Castillo I, Vizan J, Rodriguez-Sainz M, et al. An unusual mechanism for resistance to the antibiotic coumermycin A1. Proc Natl Acad Sci USA. 1991;88(19):8860-8864.

11. Holmes ML, Dyall-Smith ML. Mutations in DNA gyrase results in novobiocin resistance in halophilic archaebacteria. J Bacteriol. 1991;173(2):642-648.

12. Samuels DS, Marconi RT, Huang WM, et al. gyrB mutations in coumermycin A1-resistant Borreliaburgdorferi. $J$ Bacteriol. 1994;176(10):3072-3075.

13. Munoz R, Bustamante M, de la Campa A. Ser-127-to-Leusubstitution in the DNA gyrase B subunit of Streptococcus pneumoniaei simplicated in novobiocin resistance. J Bacteriol. 1995;177(14):4166-4170.

14. Thiara AS, Cundliffe E. Cloning and characterization of a DNA gyrase B gene from Streptomycessphaeroides that confers resistance to novobiocin. EMBO J. 1988;7(7):2255-2259.

15. Gross CH, Parsons JD, Grossman TH, et al. Active-site residues of Escherichia coli DNA gyrase required in coupling ATP hydrolysis to DNA super coiling and amino acid substitutions leading to novobiocin resistance. Antimicrob Agents Chemother. 2003;47(3):1037-1046.

16. Kampranis SC, Gormley NA, Tranter R, et al. Probing the binding of coumarins and cyclothialidines to DNA gyrase. Biochemistry. 1999;38(7):1967-1976.

17. Stieger M, Angehrn P, Wohlgensinger B, et al. GyrB mutations in Staphylococcus aureus strains resistant to cyclothialidine, coumermycin, and novobiocin. Antimicrobial agents chemotherapy. 1996;40(4):1060-1062.

18. Sanchez-Cespedes J, Saez-Lopez E, Frimodt-Moller N, et al. Effects of a mutation in the gyrA gene on the virulence of uropathogenic Escherichia coli. Antimicrobial Agents Chemotherapy. 2015;59(8):4662-4668.

19. Lafitte D, Lamour V, Tsvetkov P, et al. DNA gyrase Interaction with coumarin-based inhibitors: the role of the hydroxyl benzoate isopentenyl moiety and the 5'-methyl group of the noviose. Biochemistry. 2002;41(23):7217-7223.

20. Holdgate G, Tunnicliffe A, Ward W, et al. The entropic penalty of ordered water accounts for weaker binding of the antibiotic novobiocin to a resistant mutant of DNA gyrase: a thermodynamic and crystallographic study. Biochemistry. 1997;36(32):9663-9673.

21. Brvar M, Perdih A, Renko M, et al. Structure-based discovery of substituted 4,5'-bithiazoles as novel DNA gyrase inhibitors. $J$ Med Chem. 2012;55(14):6413-6426. 